\title{
Efficacy and safety of immunosuppressive medications for steroid-resistant nephrotic syndrome in children: a systematic review and network meta-analysis
}

\author{
Shaojun Li ${ }^{1,3}$, Haiping Yang ${ }^{2,5}$, Pengfei Guo ${ }^{1,3}$, Xiaoxiao Ao ${ }^{1,4}$, Junli Wan ${ }^{2,4}$, Qiu Li ${ }^{1,2,3}$ \\ and Liping Tan ${ }^{1,4}$ \\ ${ }^{1}$ Department of Emergency, Children's Hospital of Chongqing Medical University, Chongqing, China \\ ${ }^{2}$ Department of Nephrology, Children's Hospital of Chongqing Medical University, Chongqing, China \\ ${ }^{3}$ Ministry of Education Key Laboratory of Child Development and Disorders, Chongqing, China \\ ${ }^{4}$ Key Laboratory of Pediatrics in Chongqing, Chongqing, China \\ ${ }^{5}$ Chongqing International Science and Technology Cooperation Center for Child Development and Disorders, Chongqing, \\ China
}

Correspondence to: Liping Tan, email: tanlp0825@hotmail.com

Keywords: immunosuppressant, SRNS, pediatrics, multiple-treatments meta-analysis

Received: June 21, $2017 \quad$ Accepted: August 07, $2017 \quad$ Published: August 21, 2017

Copyright: Li et al. This is an open-access article distributed under the terms of the Creative Commons Attribution License 3.0 (CC BY 3.0 ), which permits unrestricted use, distribution, and reproduction in any medium, provided the original author and source are credited.

\section{ABSTRACT}

Background: Conventional meta-analyses and randomized controlled trials have shown inconsistent results regarding the efficacy of immunosuppressants for pediatric steroid-resistant nephrotic syndrome (SRNS).

Objective: To conduct a network meta-analysis aimed at evaluating the efficacy and safety of available immunosuppressive agents in pediatric patients with SRNS.

Study methods: MEDLINE, the Cochrane Central Register of Controlled Trials, and EMBASE were searched on January 2017. Data from randomized controlled trials (RCTs) were included. The main outcomes analyzed were efficacy [number/portion with complete remission (CR), number/portion with partial remission (PR), and total number/portion in remission (TR)] and safety [adverse secondary event (ASE) rates].

Results: A meta-analysis of 18 RCTs showed that tacrolimus was more efficacious for achieving CR than intravenous (i.v.) cyclophosphamide, mycophenolate mofetil (MMF), oral cyclophosphamide, leflunomide, chlorambucil, azathioprine, and plaebo/nontreatment (P/NT), and more efficacious than i.v. cyclophosphamide, oral cyclophosphamide, and P/NT in terms of TR outcomes. Cyclosporin was associated with a greater CR rate than i.v. cyclophosphamide, MMF, oral cyclophosphamide, chlorambucil, azathioprine, or P/NT, and associated with a greater TR rate than i.v. cyclophosphamide, oral cyclophosphamide, or P/NT. MMF was found to be more efficacious than i.v. cyclophosphamide and oral cyclophosphamide in terms of TR.

Conclusions: Tacrolimus and cyclosporine may be preferred initial treatments for children with SRNS. MMF may be another option for this patient population. Further studies of the efficacy and safety of these three drugs in children with SRNS should be pursued.

\section{INTRODUCTION}

The incidence of pediatric primary nephrotic syndrome (NS) is about $1-3 / 100,000$ children 16 years old or younger [1]. In most cases, clinical remission of primary
NS can be achieved with corticosteroid therapy [2]. The approximately $10-20 \%$ for whom complete remission is not achieved following corticosteroid therapy are classified as having steroid-resistant nephrotic syndrome (SRNS) [1]. SRNS patients are a heterogenous population 
with related diagnoses of minimal-change disease (MCD), mesangial proliferative glomerulonephritis (MesPGN), focal segmental glomerulosclerosis (FSGS), or other histopathologies [1].

Treating SRNS, which should be done under the care of a pediatric nephrologist, can be challenging because there is a paucity of strong evidence to inform SRNS treatment decisions due to the lack of largescale randomized controlled trials (RCTs). Children with SRNS may be treated with immunosuppressive agents, such as cyclosporin, cyclophosphamide, or tacrolimus [3]. Remission rates obtained with combinations of cyclophosphamide and intravenous (i.v.) methylprednisolone have reached $50-60 \%$ in observational studies and individual treatment groups in RCTs [4-7]. Meanwhile, complete remission (CR) and partial remission (PR) rates with calcineurin inhibitors (cyclosporine and tacrolimus) have been in the range of $30-80 \%$ in observational studies and RCTs [8-10]. If there is a failure to achieve at least PR, SRNS prhogresses to end-stage kidney disease $[11,12]$.

In recent decades, several new lower-toxicity immunosuppressive medications have been introduced for the treatment of SRNS in children [13]. However, these new medications have been found to be less effective for prolonging remission after corticosteroid withdrawal than traditional immunosuppressant drugs. Because head-to-head comparison trials of these new agents with traditional ones have not been completed, however, there is not a consensus regarding which immunosuppressive drugs are most suitable for treating SRNS in children Pairwise meta-analyses evaluating the efficacy of new immunosuppressive medications have identified factors that may be associated with therapeutic efficacy and previous systematic reviews have suggested efficacy differences among nonsteroidal immunosuppressive medications [14-16]. However, these studies are inconclusive because they could not provide direct comparisons. Moreover, the extent to which efficacy and safety varies across potential SRNS drugs is unclear.

Here, we report a network meta-analysis in which nine nonsteroidal immunosuppressive agents were compared with respect to efficacy and safety in children being treated for SRNS. The aim of this work was to identify a preferable SRNS therapy drug in children.

\section{RESULTS}

\section{Study characteristics and evidence network}

A total of 7,681 potentially relevant studies were retrieved, which included 6,146 non-repetitive potentially eligible articles. On the basis of our eligibility criteria (parallel RCTs whose subjects were children with initial SRNS and children with delayed SRNS and that were examining immunosuppressive medications), 6,102 articles were excluded during the title/abstract review process and, subsequently, 26 articles were excluded consequent to a full-text review. Finally, 18 articles published from 1970 to 2015 were included in our network meta-analysis. The 18 selected studies involved a total of 790 individuals who were assigned randomly to an immunosuppressive medication or placebo/nontreatment (P/NT) group. The trial selection process is summarized in Figure 1.

\section{Study characteristics}

The characteristics of the 18 included trials are summarized in Table 1 [4, 6-9, 17-29]. Briefly, trial durations ranged from 3 months to 24 months and the enrolled patients ranged in age from 1 year to 18 years old. A majority (61\%) of the participants were male. The distribution of histopathologic diagnoses in each trial are shown in Figure 2. Most $(14 / 17,82 \%)$ of the studies included patients with MCD, MesPGN, and FSGS, three studies included only patients with FSGS, and one study included only patients with MCD. Data from 790 individuals were included in the efficacy and safety analyses. The mean sample size was 44 individuals per group (range, 8-138). Most (17/18; 94\%) of the studies had two arms; one study had three arms. Regarding study quality, $67 \%$ of the trials were outcome-blinded, $56 \%$ were allocation-concealed, $50 \%$ had incomplete outcome data, and $17 \%$ were patient-blinded. Generally, the risk of bias in the reviewed trials was medium (see Supplementary Figure 1).

\section{Evidence network}

Our efficacy analyses included 730 patients with a CR in 17 trials examining a total of ten treatments (Figure 3A), 569 patients with a PR in 12 trials examining a total of seven treatments (Figure 3B), and 605 TR patients in 12 trials examining a total of seven treatments (Figure 3C). Our safety analyses based on the incidence of ASEs included 730 patients in 11 trials examining a total of seven treatments (Figure 3D). Ultimately, the following nine treatments were analyzed relative to $\mathrm{P} / \mathrm{NT}$ in the present meta-analysis: cyclosporin (7 trials), i.v. cyclophosphamide (3 trials), tacrolimus (4 trials), MMF (3 trials), oral cyclophosphamide (5 trials), leflunomide (1 trial), chlorambucil (1 trial), azathioprine (1 trials), and rituximab-cyclosporin dual therapy ( 1 trial).

\section{Conventional meta-analysis of individual medications}

The efficacy and safety analysis results obtained for individual immunosuppressive medications as determined by direct pairwise meta-analyses are shown in Figure 4. Relative to P/NT, cyclosporin was found to 
Table 1: Descriptive characteristics of studies included in the meta-analysis

\begin{tabular}{|c|c|c|c|c|c|c|c|c|c|c|c|}
\hline Study & Country & $\begin{array}{l}\text { Study } \\
\text { design }\end{array}$ & $\begin{array}{l}\text { Time } \\
\text { frame }\end{array}$ & $\begin{array}{l}\text { Cases } \\
(\mathrm{N})\end{array}$ & $\begin{array}{l}\text { Age } \\
\text { (y) }\end{array}$ & $\begin{array}{l}\text { Sex } \\
(\mathrm{M} / \mathrm{F})\end{array}$ & Patients & Interventions & Study outcomes & $\begin{array}{l}\text { Follow-up } \\
\text { duration }\end{array}$ & $\begin{array}{l}\text { Attrition } \\
(\%)\end{array}$ \\
\hline $\begin{array}{l}\text { Abramowicz } \\
1970\end{array}$ & International & $\begin{array}{l}\text { Multicenter } \\
\text { RCT }\end{array}$ & 1967-1969 & 38 & NR & $\mathrm{NR}$ & Initial SRNS & AZA vs P/NT & $\mathrm{CR}$ and $\mathrm{PR}$ at $90 \mathrm{~d}$, & 3 mos. & $18 \%$ \\
\hline $\begin{array}{l}\text { Choudhry } \\
2009\end{array}$ & India & $\begin{array}{l}\text { Single center } \\
\text { RCT }\end{array}$ & 2005-2007 & 41 & $3.5-6.0$ & $25 / 16$ & $\begin{array}{l}\text { Initial and late } \\
\text { SRNS }\end{array}$ & TAC vs CSA & $\begin{array}{l}\mathrm{CR} \text { and } \mathrm{PR} \text { at } 12 \text { mos., } \\
\text { adverse effects }\end{array}$ & 12 mos. & $0 \%$ \\
\hline D’Agati 2013 & USA & $\begin{array}{l}\text { Multicenter } \\
\text { RCT }\end{array}$ & 2004-2009 & 138 & NR & $73 / 65$ & Initial SRNS & CSA vs MMF & $\begin{array}{l}\mathrm{CR} \text { and } \mathrm{PR} \text { at } 12 \text { mos., } \\
\text { adverse effects }\end{array}$ & 12 mos. & $1 \%$ \\
\hline Elhence 1994 & India & $\begin{array}{l}\text { Single center } \\
\text { RCT }\end{array}$ & 1990-1991 & 13 & $3-16$ & $11 / 2$ & $\begin{array}{l}\text { Initial and late } \\
\text { SRNS }\end{array}$ & OCPA vs ICPA & $\begin{array}{l}\mathrm{CR} \text { at } 6 \text { mos., adverse } \\
\text { effects }\end{array}$ & $6 \mathrm{mos}$ & $15 \%$ \\
\hline Garin 1988 & USA & $\begin{array}{l}\text { Single center } \\
\text { RCT }\end{array}$ & NR & 8 & $3-18$ & $6 / 2$ & SRNS & CSA vs P/NT & CR at 3 mos. & 3 mos. & $0 \%$ \\
\hline Gulati 2012 & India & $\begin{array}{l}\text { Multicenter } \\
\text { RCT }\end{array}$ & 2008-2010 & 131 & $2-16$ & $86 / 45$ & $\begin{array}{l}\text { Initial and late } \\
\text { SRNS }\end{array}$ & TAC vs ICPA & $\begin{array}{l}\mathrm{CR} \text { and } \mathrm{PR} \text { at } 12 \text { mos., } \\
\text { adverse effects }\end{array}$ & 12 mos. & $5 \%$ \\
\hline ISKDC 1974 & International & $\begin{array}{l}\text { Multicenter } \\
\text { RCT }\end{array}$ & $1970-1972$ & 31 & NR & NR & Initial SRNS & OCPA vs P/NT & $\begin{array}{l}\mathrm{CR} \text { and } \mathrm{PR} \text { at } 12 \text { mos., } \\
\text { adverse effects }\end{array}$ & 24 mos. & $0 \%$ \\
\hline $\begin{array}{l}\text { Kleinknecht } \\
1980\end{array}$ & France & $\begin{array}{l}\text { Single center } \\
\text { RCT }\end{array}$ & NR & 30 & NR & NR & SRNS & CHL vs $\mathrm{P} / \mathrm{NT}$ & CR at 6 mos. & 6 mos. & $0 \%$ \\
\hline $\begin{array}{l}\text { Lieberman } \\
1996\end{array}$ & USA & $\begin{array}{l}\text { Multicenter } \\
\text { RCT }\end{array}$ & NR & 31 & $7-16$ & $21 / 9$ & Initial SRNS & CSA vs $\mathrm{P} / \mathrm{NT}$ & $\begin{array}{l}\mathrm{CR} \text { and PR at } 6 \text { mos., } \\
\text { adverse effects }\end{array}$ & $6 \mathrm{mos}$. & $23 \%$ \\
\hline $\begin{array}{l}\text { Magnasco } \\
2012\end{array}$ & Italy & $\begin{array}{l}\text { Single center } \\
\text { RCT }\end{array}$ & $2007-2010$ & 31 & $<16$ & $19 / 12$ & $\begin{array}{l}\text { Initial and late } \\
\text { SRNS }\end{array}$ & RTCA vs P/NT & $\begin{array}{l}\mathrm{CR} \text { at } 12 \text { mos., adverse } \\
\text { effects }\end{array}$ & 12 mos. & $0 \%$ \\
\hline Mantan 2008 & India & $\begin{array}{l}\text { Single center } \\
\text { RCT }\end{array}$ & 2001-2003 & 52 & $1-18$ & $35 / 17$ & $\begin{array}{l}\text { Initial and late } \\
\text { SRNS }\end{array}$ & ICPA vs OCPA & $\begin{array}{l}\mathrm{CR} \text { and PR at } 6 \text { mos., } \\
\text { adverse effects }\end{array}$ & 18 mos. & $6 \%$ \\
\hline Ohri 2010 & India & $\begin{array}{l}\text { Single center } \\
\text { RCT }\end{array}$ & NR & 35 & $1-12$ & $17 / 18$ & Initial SRNS & ICPA vs OCPA & $\begin{array}{l}\mathrm{CR} \text { and } \mathrm{PR} \text { at } 6 \text { mos., } \\
\text { adverse effects }\end{array}$ & $6 \mathrm{mos}$. & $0 \%$ \\
\hline Plank 2008 & International & $\begin{array}{l}\text { Multicenter } \\
\text { RCT }\end{array}$ & 2001-2004 & 32 & $1-13$ & $19 / 13$ & Initial SRNS & CSA vs CPA & $\begin{array}{l}\mathrm{CR} \text { and } \mathrm{PR} \text { at } 3 \text { mos., } \\
\text { adverse effects }\end{array}$ & 12 mos. & $33 \%$ \\
\hline $\begin{array}{l}\text { Ponticelli } \\
1993\end{array}$ & Italy & $\begin{array}{l}\text { Multicenter } \\
\text { RCT }\end{array}$ & NR & 20 & $2-18$ & NR & Initial SRNS & CSA vs $\mathrm{P} / \mathrm{NT}$ & $\begin{array}{l}\mathrm{CR} \text { and } \mathrm{PR} \text { at } 12 \text { mos., } \\
\text { adverse effects }\end{array}$ & 12 mos. & $15 \%$ \\
\hline Sinha 2015 & India & $\begin{array}{l}\text { Multicenter } \\
\text { RCT }\end{array}$ & NR & 60 & $1-18$ & NR & SRNS & TAC vs MMF & Complete or PR at 12 mos. & 12 mos. & $0.0 \%$ \\
\hline Tarshish 1996 & International & $\begin{array}{l}\text { Multicenter } \\
\text { RCT }\end{array}$ & NR & 60 & $1-16$ & NR & Initial SRNS & OCPA vs P/NT & $\begin{array}{l}\mathrm{CR} \text { and } \mathrm{PR} \text { at } 6 \text { mos., } \\
\text { adverse effects }\end{array}$ & 12 mos. & $11 \%$ \\
\hline $\begin{array}{l}\text { Valverde } \\
2010\end{array}$ & Mexico & $\begin{array}{l}\text { Single center } \\
\text { RCT }\end{array}$ & NR & 17 & $1-18$ & NR & SRNS & CSA vs TAC & $\begin{array}{l}\mathrm{CR} \text { and } \mathrm{PR} \text { at } 12 \text { mos., } \\
\text { adverse effects }\end{array}$ & 12 mos. & $0 \%$ \\
\hline Wu 2015 & China & $\begin{array}{l}\text { Single center } \\
\text { RCT }\end{array}$ & 2008-2012 & 18 & $2-18$ & $11 / 7$ & SRNS & $\begin{array}{l}\text { CPA vs MMF vs } \\
\text { LEF }\end{array}$ & CR at 6 mos. & 12 mos. & $18 \%$ \\
\hline
\end{tabular}

Notes: SRNS, steroid-resistant nephrotic syndrome; NR, not reported; RCT, randomized controlled trial; CSA, cyclosporin; ICPA, intravenous cyclophosphamide; TAC, tacrolimus, MMF, mycophenolate mofetil; OCPA, oral cyclophosphamide; LEF, leflunomide; CHL, chlorambucil; AZA, azathioprine; RTCA, rituximab-cyclosporin dual therapy; P/NT, placebo/nontreatment; CR, complete response; PR, partial response.

have significantly better efficacy for achieving both CR and PR (both, $\mathrm{I}^{2}=0 \%$ ). Cyclosporin also showed better efficacy than i.v. cyclophosphamide for both PR and TR outcomes (both $\mathrm{I}^{2}=0 \%$ ), as well as greater efficacy than MMF for TR $\left(\mathrm{I}^{2}=0 \%\right)$, though in the latter case the benefits were less clear $(95 \%$ CI for OR slightly more than 1). Additionally, tacrolimus was more efficacious than i.v. cyclophosphamide for TR $\left(\mathrm{I}^{2}=0 \%\right)$ and i.v. cyclophosphamide was more efficacious than oral cyclophosphamide for CR $\left(\mathrm{I}^{2}=37.8 \%\right)$.

In terms of ASEs, oral cyclophosphamide was found to be safer than i.v. cyclophosphamide (95\% CI for OR slightly more than 1) and tacrolimus was found to be safer than both cyclosporin and i.v. cyclophosphamide $\left(\mathrm{I}^{2}=15.1 \%\right)$. There were no significant differences in safety revealed by other direct comparisons between 
immunosuppressants. However, it should be noted that the $95 \%$ CIs obtained for most of the comparisons were reflective of either high or no heterogeneity due to the small number of studies included in the pairwise comparisons; overall, heterogeneity was moderate.

\section{Network meta-analysis of individual medications}

Our network meta-analysis results for immunosuppressive medications, active comparators, and $\mathrm{P} / \mathrm{NT}$ are presented in Figure 4. Tacrolimus was found to be more efficacious for achieving CR than i.v. cyclophosphamide, MMF, oral cyclophosphamide, leflunomide, chlorambucil, and azathioprine, as well as P/NT. Tacrolimus was also found to be more efficacious for achieving TR than i.v. cyclophosphamide, oral cyclophosphamide, azathioprine, and P/NT. Meanwhile, cyclosporin therapy was associated with a better $\mathrm{CR}$ rate than i.v. cyclophosphamide, $\mathrm{MMF}$, oral cyclophosphamide, chlorambucil, azathioprine, or P/NT. Cyclosporin was more likely to yield more TR outcomes than i.v. cyclophosphamide, oral cyclophosphamide,
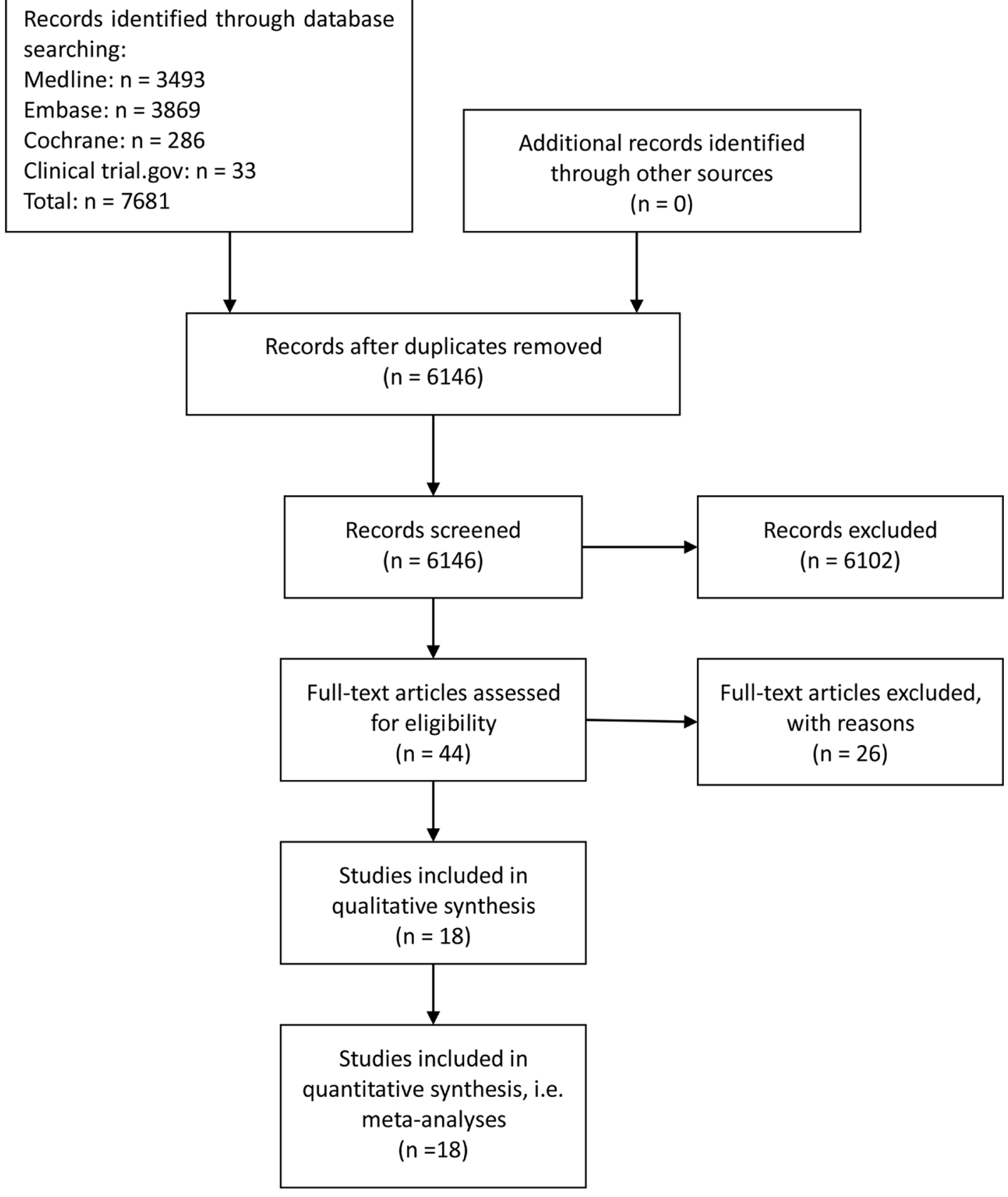

Additional records identified through other sources $(n=0)$

Figure 1: Flowchart of included studies. 


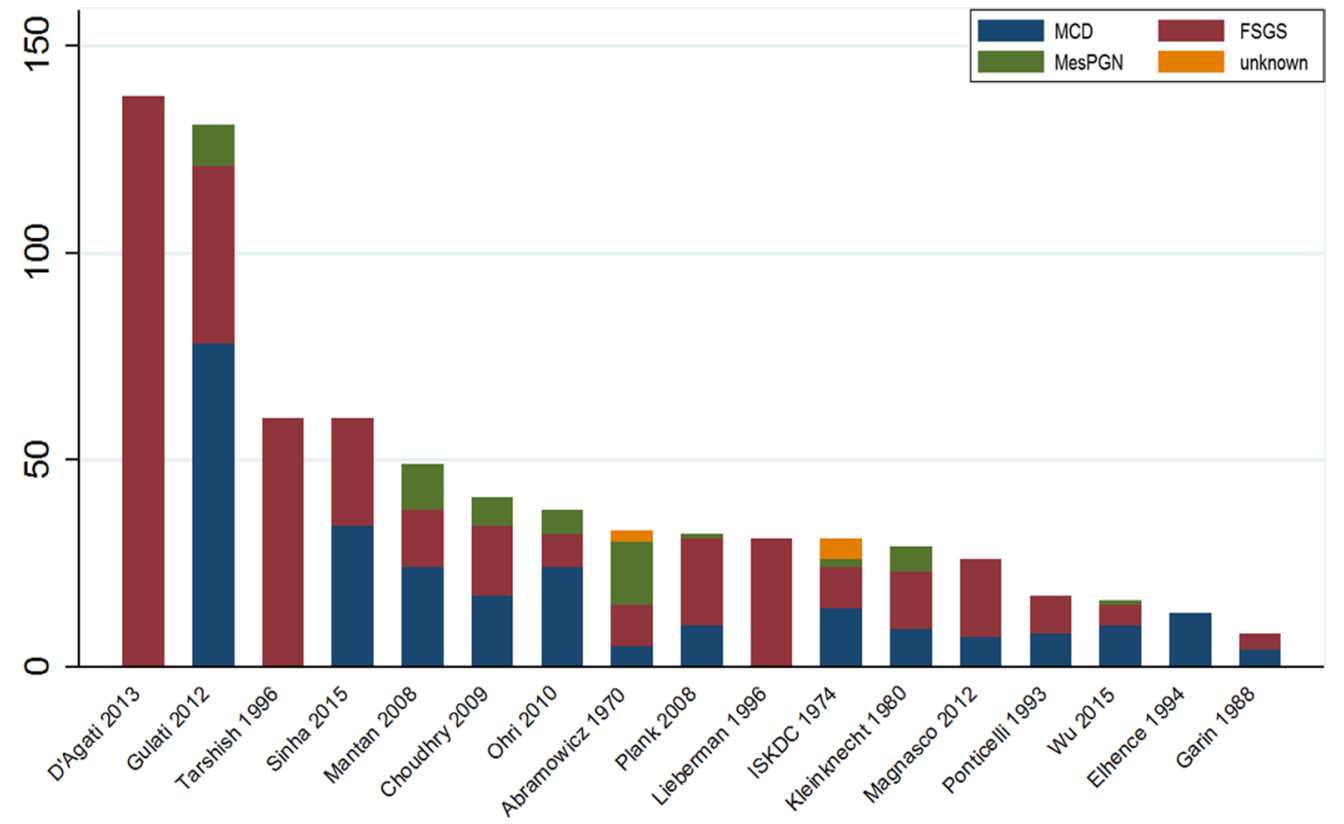

Figure 2: Distribution of histopathologic diagnoses in included RCTs.

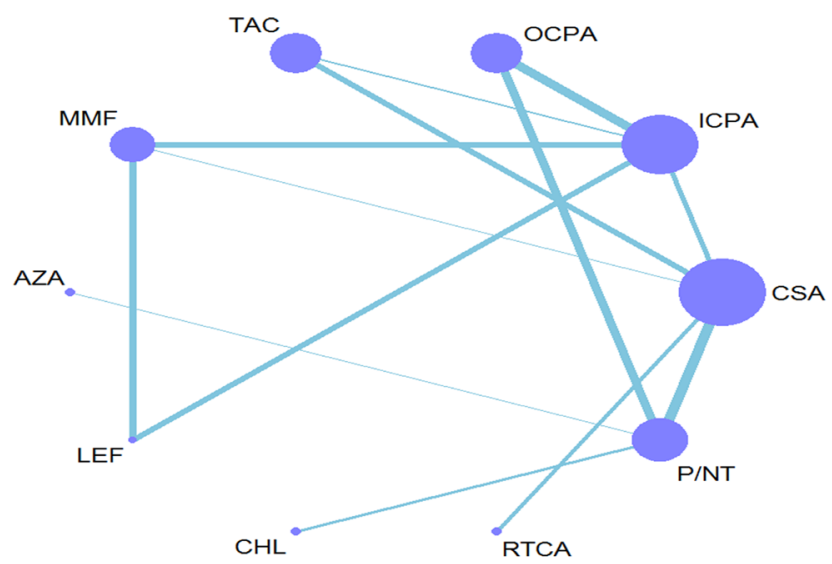

A. Complete remission

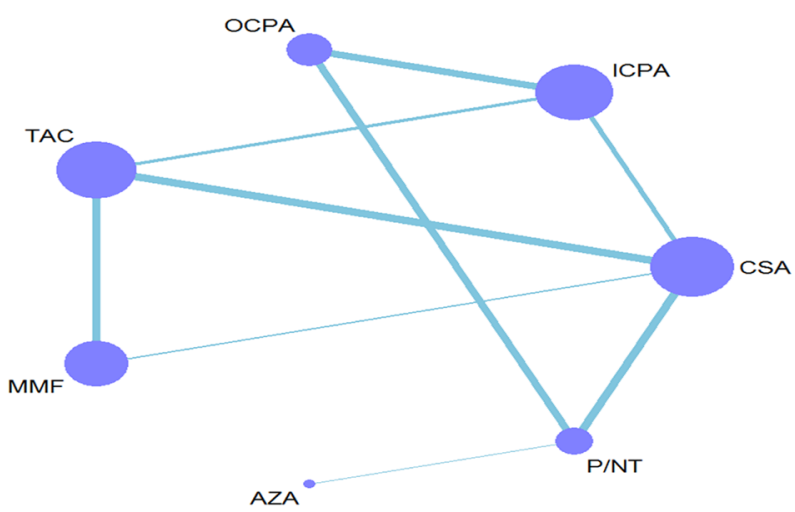

C. Total remission

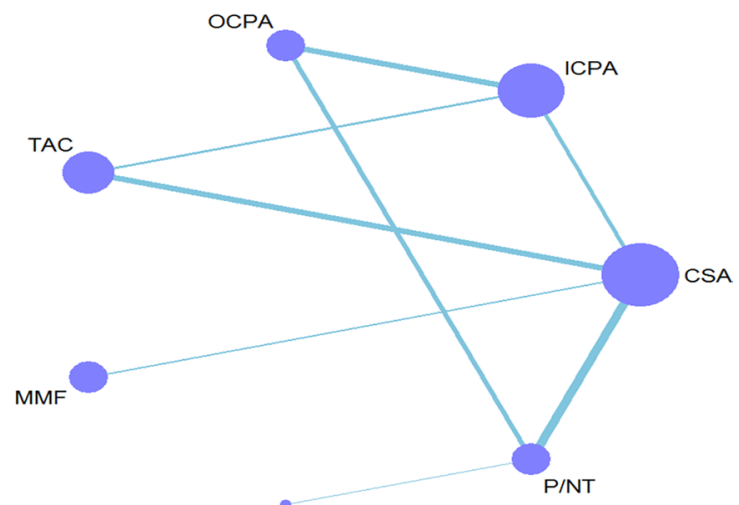

$\mathrm{AZA}$

B. Partial remission

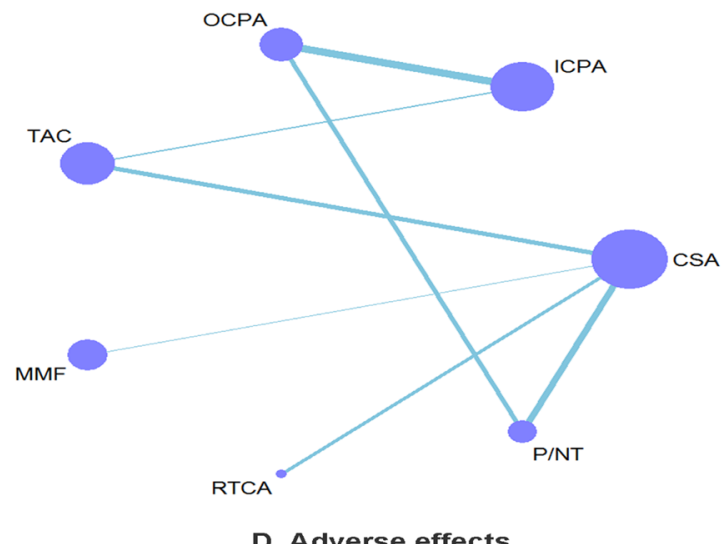

D. Adverse effects

Figure 3: Network of eligible efficacy and safety comparisons. (A-D) The thickness of the lines reflects the number of studies being compared, and node size reflects the number of individuals treated with each pharmacotherapy. 


\begin{tabular}{|c|c|c|c|c|c|c|c|c|c|}
\hline AZA & NA & $\begin{array}{c}2.41 \\
(0.87,6.71)\end{array}$ & NA & NA & NA & NA & $\begin{array}{c}1.08 \\
(0.13,8.80)\end{array}$ & NA & NA \\
\hline $\begin{array}{c}0.93 \\
(0.07,11.99)\end{array}$ & CHL & NA & NA & NA & NA & NA & $\begin{array}{c}1.00 \\
(0.23,4.31)\end{array}$ & NA & NA \\
\hline$\frac{0.11}{(0.01,1.23)}$ & $\frac{0.11}{(0.02,0.79)}$ & CSA & $\begin{array}{c}0.41 \\
(0.03,5.00)\end{array}$ & NA & NA & NA & $\frac{0.15}{(0.02,1.00)}$ & $\begin{array}{c}0.92 \\
(0.16,5.49)\end{array}$ & $\begin{array}{c}0.60 \\
(0.20,1.80)\end{array}$ \\
\hline $\begin{array}{c}0.56 \\
(0.05,5.97)\end{array}$ & $\begin{array}{c}0.60 \\
(0.10,3.74)\end{array}$ & $\frac{5.26}{(1.94,14.24)}$ & ICPA & $\begin{array}{c}0.40 \\
(0.04,3.96)\end{array}$ & $\begin{array}{c}0.67 \\
(0.06,7.35)\end{array}$ & $\frac{0.58}{(0.25,1.33)}$ & NA & NA & $\begin{array}{c}6.36 \\
(2.68,15.07)\end{array}$ \\
\hline $\begin{array}{c}0.90 \\
(0.04,20.53)\end{array}$ & $\begin{array}{c}0.96 \\
(0.06,15.01)\end{array}$ & $\begin{array}{c}8.42 \\
(0.97,73.11)\end{array}$ & $\begin{array}{c}1.60 \\
(0.20,13.13)\end{array}$ & LEF & $\begin{array}{c}1.67 \\
(0.15,18.87)\end{array}$ & NA & NA & NA & NA \\
\hline $\begin{array}{c}0.31 \\
(0.02,4.10)\end{array}$ & $\begin{array}{c}0.33 \\
(0.04,2.72)\end{array}$ & $\frac{2.89}{(1.12,7.51)}$ & $\begin{array}{c}0.55 \\
(0.16,1.95)\end{array}$ & $\begin{array}{c}0.34 \\
(0.04,2.94)\end{array}$ & MMF & NA & NA & NA & NA \\
\hline $\begin{array}{c}0.93 \\
(0.10,9.02)\end{array}$ & $\begin{array}{c}1.00 \\
(0.18,5.48)\end{array}$ & $\frac{\stackrel{8.73}{(2.72,27.96)}}{}$ & $\begin{array}{c}1.66 \\
(0.75,3.66)\end{array}$ & $\begin{array}{c}1.04 \\
(0.11,9.60)\end{array}$ & $\begin{array}{c}3.01 \\
(0.73,12.40)\end{array}$ & OCPA & $\begin{array}{c}0.94 \\
(0.37,2.41)\end{array}$ & NA & NA \\
\hline $\begin{array}{c}0.93 \\
(0.11,7.59)\end{array}$ & $\begin{array}{c}1.00 \\
(0.23,4.31)\end{array}$ & $\frac{8.73}{(2.47,30.88)}$ & $\begin{array}{c}1.66 \\
(0.56,4.96)\end{array}$ & $\begin{array}{c}1.04 \\
(0.10,10.60)\end{array}$ & $\begin{array}{c}3.02 \\
(0.66,13.70)\end{array}$ & $\begin{array}{c}1.00 \\
(0.42,2.39)\end{array}$ & PLA & NA & NA \\
\hline $\begin{array}{c}0.12 \\
(0.01,2.39)\end{array}$ & $\begin{array}{c}0.12 \\
(0.01,1.72)\end{array}$ & $\begin{array}{c}1.08 \\
(0.18,6.44)\end{array}$ & $\begin{array}{c}0.21 \\
(0.03,1.59)\end{array}$ & $\begin{array}{c}0.13 \\
(0.01,2.12)\end{array}$ & $\begin{array}{c}0.37 \\
(0.05,2.82)\end{array}$ & $\frac{0.12}{(0.01,1.04)}$ & $\frac{0.12}{(0.01,1.10)}$ & RTCA & NA \\
\hline 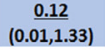 & 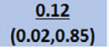 & $\begin{array}{c}1.08 \\
(0.43,2.71)\end{array}$ & $\frac{\underline{0.21}}{(0.09,0.45)}$ & $\frac{0.13}{(0.01,1.13)}$ & $\begin{array}{c}0.37 \\
(0.11,1.30)\end{array}$ & $\frac{0.12}{(0.04,0.36)}$ & $\frac{0.12}{(0.04,0.43)}$ & $\begin{array}{c}1.00 \\
(0.13,7.41)\end{array}$ & TAC \\
\hline
\end{tabular}

\section{A. OR with $95 \% \mathrm{Cl}$ of network meta-analysis for CR}

\begin{tabular}{|c|c|c|c|c|c|c|}
\hline AZA & NA & NA & NA & NA & $\begin{array}{c}1.08 \\
(0.13,8.80)\end{array}$ & NA \\
\hline $\begin{array}{c}0.28 \\
(0.01,6.83)\end{array}$ & CSA & $\begin{array}{c}0.15 \\
(0.03,0.91)\end{array}$ & $\begin{array}{c}0.89 \\
(0.41,1.93)\end{array}$ & NA & $\begin{array}{c}0.17 \\
(0.04,0.77)\end{array}$ & $\begin{array}{c}2.77 \\
(0.82,9.40)\end{array}$ \\
\hline $\begin{array}{c}0.66 \\
(0.02,18.63)\end{array}$ & $\begin{array}{c}2.36 \\
(0.47,11.79)\end{array}$ & ICPA & NA & $\begin{array}{c}1.64 \\
(0.42,6.33)\end{array}$ & NA & $\begin{array}{c}0.96 \\
(0.45,2.05)\end{array}$ \\
\hline $\begin{array}{c}0.31 \\
(0.01,12.90)\end{array}$ & $\begin{array}{c}1.12 \\
(0.17,7.48)\end{array}$ & $\begin{array}{c}0.47 \\
(0.04,5.71)\end{array}$ & MMF & NA & NA & NA \\
\hline $\begin{array}{c}0.63 \\
(0.02,16.23)\end{array}$ & $\begin{array}{c}2.24 \\
(0.33,15.16)\end{array}$ & $\begin{array}{c}0.95 \\
(0.19,4.77)\end{array}$ & $\begin{array}{c}2.00 \\
(0.13,29.58)\end{array}$ & ОСРА & $\begin{array}{c}1.20 \\
(0.35,4.15)\end{array}$ & NA \\
\hline $\begin{array}{c}0.93 \\
(0.06,14.16)\end{array}$ & $\begin{array}{c}3.31 \\
(0.63,17.42)\end{array}$ & $\begin{array}{c}1.40 \\
(0.20,9.58)\end{array}$ & $\begin{array}{c}2.95 \\
(0.24,36.80)\end{array}$ & $\begin{array}{c}1.48 \\
(0.25,8.72)\end{array}$ & PLA & NA \\
\hline $\begin{array}{c}0.24 \\
(0.01,7.61)\end{array}$ & $\begin{array}{c}0.87 \\
(0.19,4.02)\end{array}$ & $\begin{array}{c}0.37 \\
(0.07,1.88)\end{array}$ & $\begin{array}{c}0.78 \\
(0.07,8.90)\end{array}$ & $\begin{array}{c}0.39 \\
(0.05,3.13)\end{array}$ & $\begin{array}{c}0.26 \\
(0.03,2.14)\end{array}$ & TAC \\
\hline
\end{tabular}

\section{B. OR with $95 \% \mathrm{Cl}$ of network meta-analysis for PR}

\begin{tabular}{|c|c|c|c|c|c|c|}
\hline AZA & NA & NA & NA & NA & $\begin{array}{c}1.09 \\
(0.22,5.45)\end{array}$ & NA \\
\hline $\begin{array}{c}0.17 \\
(0.02,1.48)\end{array}$ & CSA & $\begin{array}{c}0.14 \\
(0.03,0.72)\end{array}$ & $\begin{array}{c}0.59 \\
(0.30,1.18)\end{array}$ & NA & $\begin{array}{c}0.14 \\
(0.01,1.68)\end{array}$ & $\begin{array}{c}1.74 \\
(0.40,7.63)\end{array}$ \\
\hline $\begin{array}{c}0.95 \\
(0.12,7.30)\end{array}$ & $\begin{array}{c}5.48 \\
(2.15,13.99)\end{array}$ & ICPA & NA & $\begin{array}{c}0.86 \\
(0.35,2.08)\end{array}$ & NA & $\begin{array}{c}5.57 \\
(2.45,12.68)\end{array}$ \\
\hline $\begin{array}{c}0.28 \\
(0.03,2.52)\end{array}$ & $\begin{array}{c}1.64 \\
(0.88,3.08)\end{array}$ & $\begin{array}{c}0.30 \\
(0.11,0.80)\end{array}$ & MMF & NA & NA & $\begin{array}{c}1.72 \\
(0.62,4.79)\end{array}$ \\
\hline $\begin{array}{c}1.15 \\
(0.17,7.79)\end{array}$ & $\begin{array}{c}6.65 \\
(2.01,21.98)\end{array}$ & $\begin{array}{c}1.21 \\
(0.52,2.83)\end{array}$ & $\begin{array}{c}4.05 \\
(1.17,14.00)\end{array}$ & OCPA & $\begin{array}{c}1.33 \\
(0.44,4.04)\end{array}$ & NA \\
\hline $\begin{array}{c}0.92 \\
(0.18,4.58)\end{array}$ & $\begin{array}{c}5.29 \\
(1.28,21.91)\end{array}$ & $\begin{array}{c}0.97 \\
(0.28,3.38)\end{array}$ & $\begin{array}{c}3.22 \\
(0.74,14.08)\end{array}$ & $\begin{array}{c}0.80 \\
(0.28,2.23)\end{array}$ & PLA & NA \\
\hline $\begin{array}{c}0.15 \\
(0.02,1.30)\end{array}$ & $\begin{array}{c}0.89 \\
(0.39,2.03)\end{array}$ & $\begin{array}{c}0.16 \\
(0.08,0.34)\end{array}$ & $\begin{array}{c}0.54 \\
(0.24,1.22)\end{array}$ & $\begin{array}{c}0.13 \\
(0.05,0.40)\end{array}$ & $\begin{array}{c}0.17 \\
(0.04,0.68)\end{array}$ & TAC \\
\hline
\end{tabular}

\section{OR with $95 \% \mathrm{Cl}$ of network meta-analysis for TR}

\begin{tabular}{|c|c|c|c|c|c|c|}
\hline CSA & NA & $\begin{array}{c}1.35 \\
(0.57,3.17)\end{array}$ & NA & $\begin{array}{c}1.35 \\
(0.31,5.86)\end{array}$ & $\begin{array}{c}19.20 \\
(0.97,378.28)\end{array}$ & $\frac{\underline{0.25}}{(0.08,0.80)}$ \\
\hline $\begin{array}{c}0.91 \\
(0.21,3.92)\end{array}$ & ICPA & NA & $\begin{array}{c}0.4 \\
6(0.20,1.09)\end{array}$ & NA & NA & $\frac{\underline{0.38}}{(0.16,0.93)}$ \\
\hline $\begin{array}{c}0.74 \\
(0.23,2.40)\end{array}$ & $\begin{array}{c}0.82 \\
(0.13,5.35)\end{array}$ & MMF & NA & NA & NA & NA \\
\hline $\begin{array}{c}1.71 \\
(0.37,8.00)\end{array}$ & $\begin{array}{c}1.89 \\
(0.72,4.94)\end{array}$ & $\begin{array}{c}2.31 \\
(0.33,15.98)\end{array}$ & OCPA & NA & $\begin{array}{c}0.82 \\
(0.18,3.79)\end{array}$ & NA \\
\hline $\begin{array}{c}1.19 \\
(0.30,4.66)\end{array}$ & $\begin{array}{c}1.31 \\
(0.28,6.07)\end{array}$ & $\begin{array}{c}1.60 \\
(0.27,9.68)\end{array}$ & $\begin{array}{c}0.69 \\
(0.16,2.95)\end{array}$ & PLA & NA & NA \\
\hline$\frac{0.05}{(0.00,1.14)}$ & $\begin{array}{c}0.06 \\
(0.00,1.75)\end{array}$ & $\begin{array}{c}0.07 \\
(0.00,1.91)\end{array}$ & $\frac{0.03}{(0.00,0.96)}$ & $\frac{0.04}{(0.00,1.28)}$ & RTCA & NA \\
\hline $\begin{array}{c}3.10 \\
(0.88,10.94)\end{array}$ & $\frac{3.42}{(1.02,11.50)}$ & $\begin{array}{c}4.18 \\
(0.75,23.35)\end{array}$ & $\begin{array}{c}1.81 \\
(0.45,7.30)\end{array}$ & $\begin{array}{c}2.61 \\
(0.51,13.24)\end{array}$ & $\begin{array}{c}59.56 \\
(2.12,1670.83)\end{array}$ & TAC \\
\hline
\end{tabular}

\section{OR with $95 \% \mathrm{Cl}$ of network meta-analysis for ASE rate}

Note: The treatments are reported in alphabetical order. The results of direct comparisons and network meta-analysis are reported above and below the diagonal division, respectively. For direct comparisons, ORs > 1 favor the column-defining treatment. For network meta-analyses, ORs $>1$ favor the first drug in alphabetical order. Significant comparisons are underscored and bolded. CSA, cyclosporin; ICPA, intravenous cyclophosphamide; TAC, tacrolimus; MMF, mycophenolate mofetil; OCPA, oral cyclophosphamide; LEF, leflunomide; CHL, chlorambucil; AZA, azathioprine; RTCA, rituximab-cyclosporin dual therapy; and P/NT, placebo/nontreatment.

Figure 4: Comparison of efficacy across drugs. OR with $95 \% \mathrm{CI}$ of network meta-analysis for CR (A), PR (B), TR (C) and ASE (D). 
or P/NT. Additionally, rituximab-cyclosporin dual therapy was more efficacious for obtaining CR than oral cyclophosphamide or P/NT, and MMF more efficacious in terms of TR than either i.v. cyclophosphamide or oral cyclophosphamide. However, in terms of safety, tacrolimus use was less likely to result in ASEs than i.v. cyclophosphamide or rituximab-cyclosporin dual therapy. Meanwhile, rituximab-cyclosporin dual therapy was associated with a greater likelihood of ASEs than oral cyclophosphamide, cyclosporin, or P/NT (95\% CI for OR slightly more than 1).

\section{Ranking of medications}

The relative efficacy and safety rankings of the interventions are shown in Figure 5. Cyclosporin, tacrolimus, rituximab-cyclosporin dual therapy, and MMF were among the most efficacious treatments for achieving CR (Figure 5A). The cumulative probabilities of CR for the examined medications were: cyclosporin $(88.7 \%)$, tacrolimus $(86.4 \%)$, rituximab-cyclosporin (82.8\%), MMF (59.8\%), i.v. cyclophosphamide (44.8\%), leflunomide
(31.5\%), chlorambucil (28.6\%), azathioprine (28.6\%), P/NT $(24.5 \%)$, and oral cyclophosphamide $(24.2 \%)$. Tacrolimus, cyclosporin, and MMF were the most efficacious treatments for achieving of PR (Figure 5B), and the cumulative probabilities of the analyzed pharamcotherapies being the most efficacious medication were: tacrolimus (74.1\%), cyclosporin (71.7\%), MMF (65.9\%), oral cyclophosphamide (41.1\%), i.v. cyclophosphamide $(37.6 \%)$, azathioprine (33.5\%), and P/NT (26.1\%). Finally, for TR, tacrolimus, cyclosporin, and MMF were the most efficacious treatments (Figure 5C), and the cumulative probabilities of each treatment being the most efficacious medication were: tacrolimus (91.5\%), cyclosporin (87.8\%), MMF (65.7\%), P/NT (29.2\%), i.v. cyclophosphamide (28.1\%), azathioprine (28.1\%) and oral cyclophosphamide (19.6\%). In terms of safety, rituximab-cyclosporin dual therapy was the treatment that was most likely to produce ASEs (Figure 5D), and the cumulative probabilities of being the most adverse medication were: rituximab-cyclosporin (96.8\%), MMF $(62.8 \%)$, intravenous cyclophosphamide (58.3\%), cyclosporin (52.4\%), P/NT (44.8\%), oral cyclophosphamide $(27.8 \%)$, and tacrolimus $(7.3 \%)$.

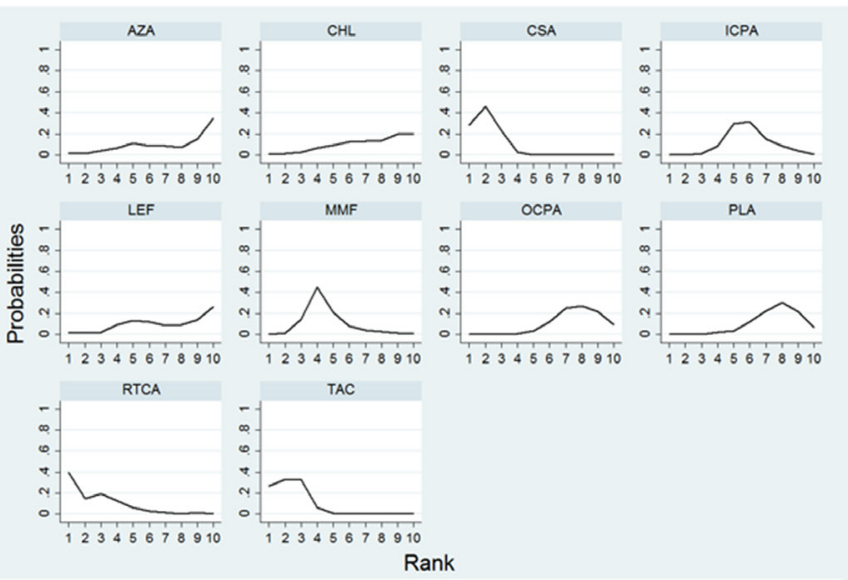

A. Rank of CR
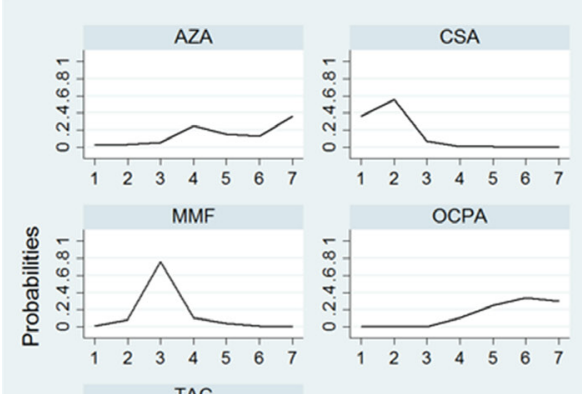

OCPA
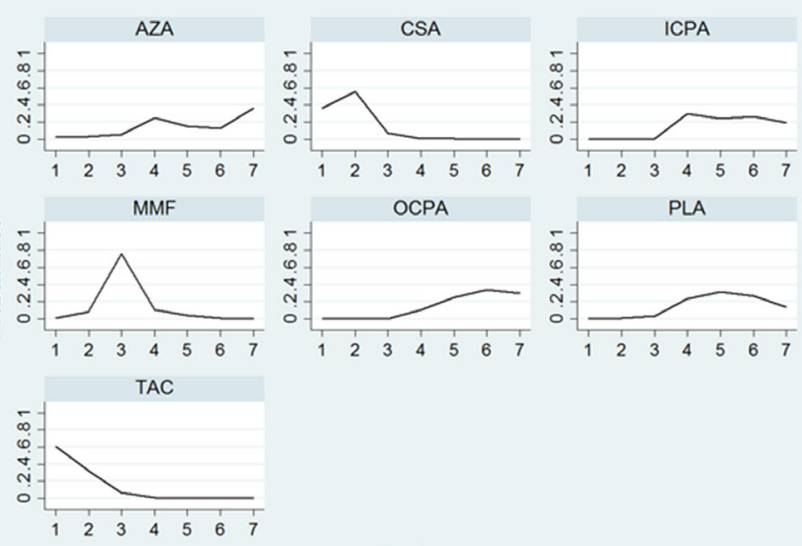

Rank

C. Rank of PR
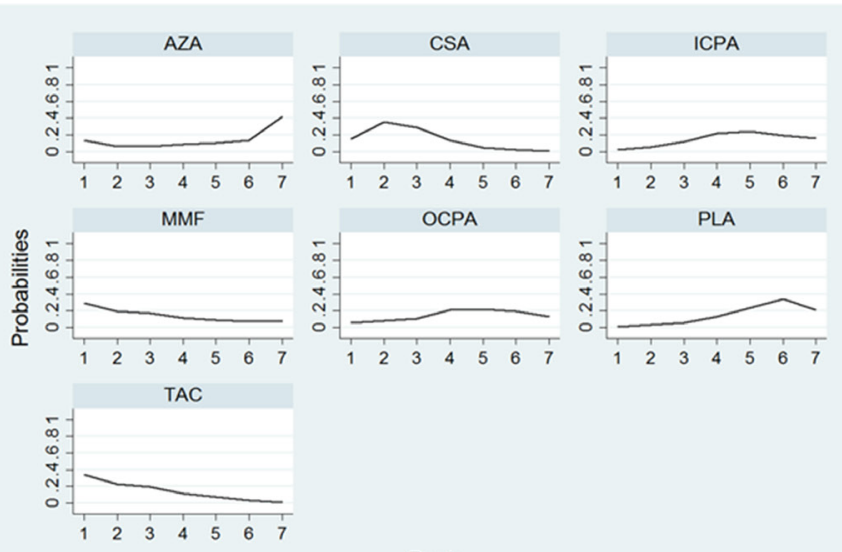

Rank

B. Rank of CR
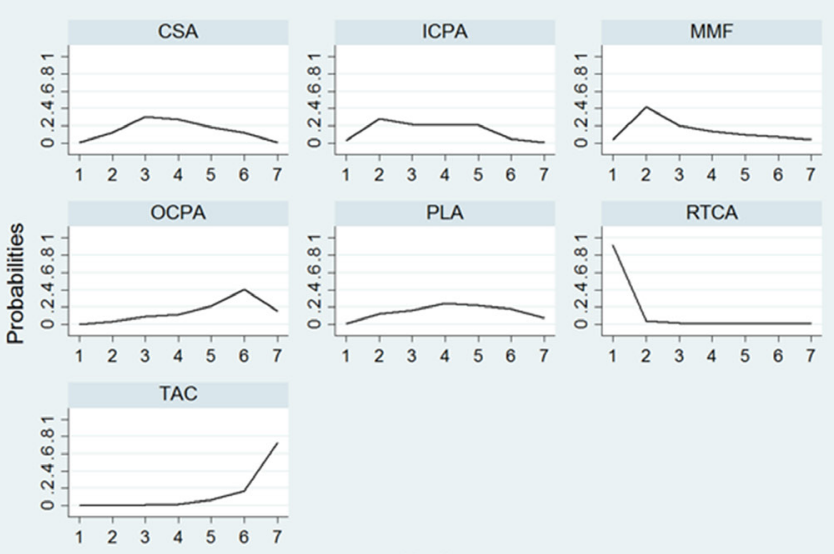

D. Rank of ASE

Figure 5: Efficacy and safety outcome rankings. CR (A), PR (B), TR (C) and ASE (D) rankings reflect the probability of being the best, second best, etc., treatment among the treatments compared. 


\section{Inconsistency and publication bias}

Inconsistency between direct and indirect comparisons of recurrence rates was low (Figure 6). Most loops (networks of three or four comparisons that arise when collating studies involving different treatments) were consistent, with the $95 \%$ CIs for the inconsistency factor (including 0) indicating similar effect estimations for

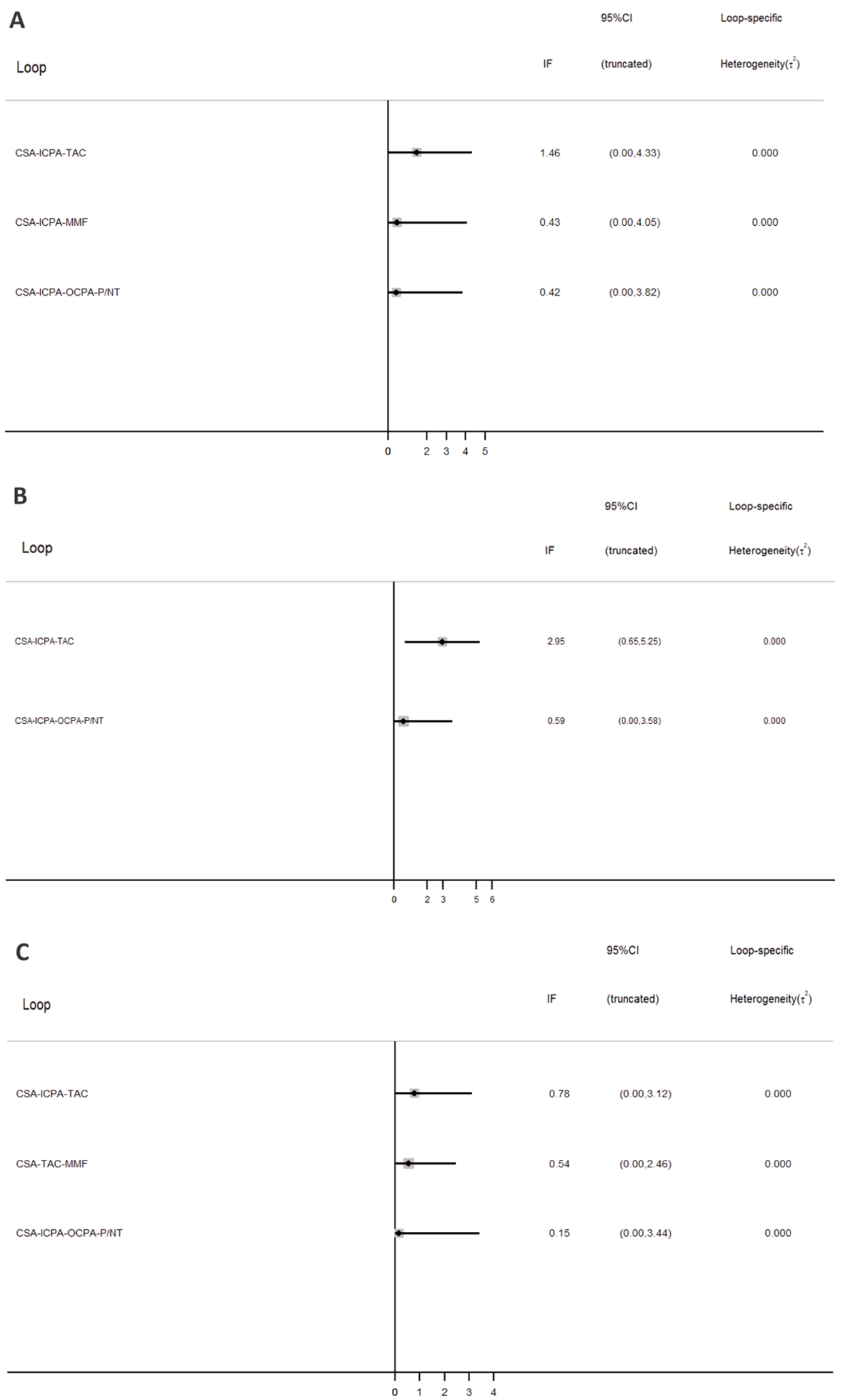

Figure 6: Inconsistency in closed loops at CR (A) PR (B) and TR (C). Graph shows estimates of differences between direct and indirect estimates as represented by $95 \%$ CIs. 
direct and indirect comparisons. Hence, the network metaanalysis results can be considered robust. Comparisonadjusted funnel plots for CR outcomes show no evidence of asymmetry (Figure 7).

\section{DISCUSSION}

In the present meta-analysis of 18 trials, with 790 individuals diagnosed with SRNS assigned randomly to one of nine immunosuppressive medication groups or a $\mathrm{P} / \mathrm{NT}$ group, we found that tacrolimus was more efficacious for achieving $\mathrm{CR}$ or TR than i.v. cyclophosphamide, MMF, oral cyclophosphamide, leflunomide, chlorambucil, azathioprine, and P/NT, with a lower ASE risk than i.v. cyclophosphamide or rituximab-cyclosporin dual therapy. Cyclosporin also fared well, being associated with greater likelihood of CR or TR than intravenous cyclophosphamide, MMF, oral cyclophosphamide, chlorambucil, azathioprine, or $\mathrm{P} / \mathrm{NT}$. Hence, of the nine immunosuppressive pharmacotherapies analyzed, tacrolimus and cyclosporin emerged as the two most efficacious agents while maintaining relatively low
ASE risk levels. A practical implication of our results is that tacrolimus and cyclosporin should be favored as firstline treatments for pediatric patients experiencing SRNS owing to their high efficacy and generally good, albeit not superior, safety. Given that tacrolimus had a similar efficacy but lower ASE likelihood than cyclosporin, further well designed RCTs are needed to evaluate the relative benefits and harms of tacrolimus versus cyclosporin for the treatment of SRNS in children.

Although MMF ranked favorably in efficacy and safety, the MMF data lacked power in the network metaanalysis indicating that there is a need for additional studies of the efficacy and safety of MMF in children with SRNS. The efficacy and safety outcomes for cyclophosphamide therapies were mediocre. Notwithstanding, it is worth noting that our analysis showed greater efficacy, but lesser safety, for i.v. cyclophosphamide relative to oral cyclophosphamide. Hence, the present results do not support the use of cyclophosphamide as a firstline treatment for SRNS. Finally, although rituximabcyclosporin dual therapy was found to have a somewhat favorable CR outcome likelihood, especially compared

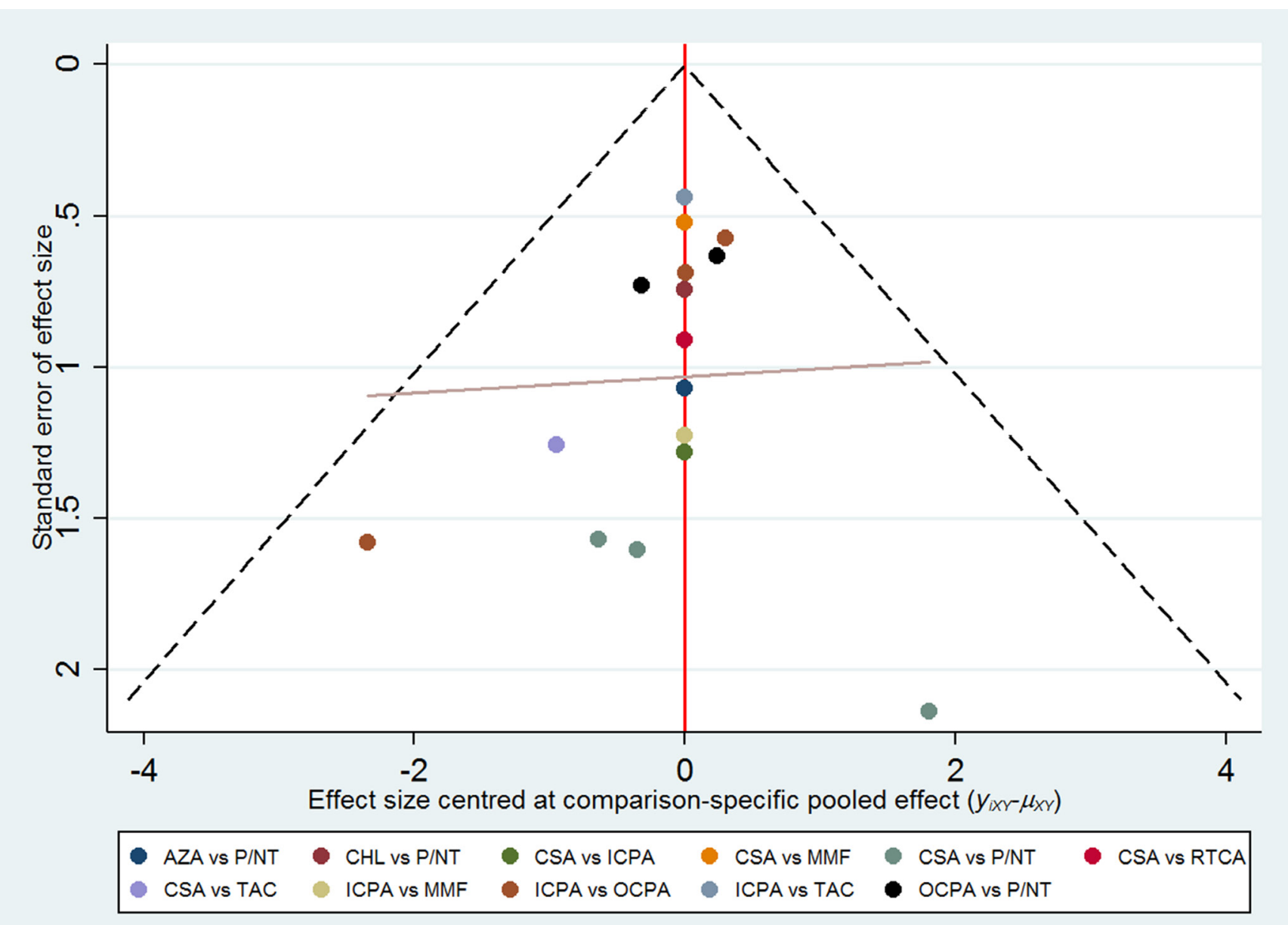

Figure 7: Summary of comparison-adjusted funnel plot results for CR rate. 
with oral cyclophosphamide, it had the poorest safety outcome of all of the regimens examined.

Our findings, which indicate clinically important differences in efficacy and safety among the examined drugs, provide reference information that can be used in immunosuppressive medication selection for treatment of SRNS in pediatric patients. Previous conventional pairwise meta-analyses of the efficacy of immunosuppressive medications for SRNS in children were inconclusive due to limitations in treatment effect information and failures to demonstrate clear relative efficacy benefits of particular drugs [15, 16, 30, 31]. Notwithstanding, a previous systematic review contributed by Hodson et al. indicated that calcineurin inhibitors yield better rates of CR or PR than P/NT or cyclophosphamide in children with SRNS [30].

The present study provides much needed direct comparisons between immunosuppressive agents in patients recovering from primary NS. The 2015 clinical guidelines for pediatric idiopathic NS recommended cyclosporine as a first-line treatment for SRNS (recommendation grade A). The guidelines suggested that tacrolimus be considered as a treatment option for patients with SRNS when cyclosporine is counter-indicated due to cosmetic side effects (recommendation grade $\mathrm{C} 1$ ), and that cyclophosphamide not be considered for induction therapy in children with SRNS (recommendation grade C2) [32].

The most problematic ASE of long-term cyclosporine use is chronic nephrotoxicity, an increased risk for which has been associated with cyclosporine treatment for $\geq 2$ years [33-35]. The number of serious ASEs of immunosuppressive agents reported here may be an underestimate because the analyzed studies were not designed primarily to evaluate harm. Additionally, because we did not exclude trials with combined corticosteroid regimens, our findings related to immunosuppressive agents cannot be considered independent of potential corticosteroid effects. Our results should not be generalized to patients who exhibit corticosteroid dependence because we did not include trials with that patient population.

The results of this analysis apply to treatment periods of 2 years or less. Longer term clinical efficacy and safety beyond 2 years may differ substantially from outcomes recorded within 2 years [35]. Additionally, the quality of our analysis may be limited by the quality of the original data. Many of the studies included in our review did not report adequate information on allocation concealment and randomization, which could influence the overall validity of the data [36]. Only $17 \%$ of the examined studies had low performance risk. We did not conduct publication bias or subgroup analyses due to the small numbers of studies examining each medication. The small number and small sample sizes of the included studies could also be of concern for the generalizability of our results. Finally, none of the included studies addressed fertility-related complications of alkylating agent therapy.

This study focused on examining RCTs of available immunosuppressive agents in pediatric SRNS patients. Recently, ongoing or completed small sample case series of new biologics - including anti-CD20 ofatumumab, abatacept, adalimumab, fresolimumab, and saquinavir which target immune cell subsets or activation pathways selectively - have spurred a new direction of hypothesisdriven therapies that may improve outcomes of children with kidney disease $[37,38]$. Further research should be conducted to determine the benefits and risks of these therapies in children with SRNS.

In conclusion, on the basis of all available direct and indirect evidence, our results suggest that tacrolimus and cyclosporine are preferable first-line medications for initial SRNS in children. MMF may be an acceptable option for patients with SRNS. Further studies are needed to evaluate the relative benefits and harms of tacrolimus versus cyclosporin for pediatric SRNS treatment. Additional information about the safety and efficacy of MMF in children with SRNS is also needed.

\section{MATERIALS AND METHODS}

\section{Identification of trials}

In preparation for this network meta-analysis, we drafted a study protocol and published it on the PROSPERO website (CRD42017062564). Clinical trials comparing at least two different treatments were searched in MEDLINE (1950 to January 2017), the Cochrane Central Register of Controlled Trials (CENTRAL, The Cochrane Library, Issue 7, 2017), and EMBASE (1974 to January 2017) with the following search terms: "immunosuppressive agents" or "alkylating agents" or "azathioprine" or "cyclosporine" or "cyclophosphamide" or "mycophenolic acid" or "rituximab" or "chlorambucil" or "levamisole" or "tacrolimus"; and "nephrotic syndrome" or "minimal change nephrotic syndrome" or "glomerulonephritis membranoproliferative" or "focal segment glomerulosclerosis" or "membranoproliferative glomerulonephritis". The search results were restricted to articles reporting studies involving children. Additionally, the reference lists of all included publications and relevant reviews were screened and ClinicalTrials.gov was searched for trials in progress.

\section{Selection criteria}

Parallel RCTs in which children with initial SRNS and children with delayed SRNS were the subjects and comprehensive comparisons of any of the following agents were included: cyclophosphamide, cyclosporine, 
tacrolimus, mycophenolate mofetil (MMF), azathioprine, chlorambucil, rituximab, or levamisole. The experimental interventions could be compared to a placebo/nontreatment (P/NT) and/or another immunosuppressive medication. SRNS was defined as persistence of proteinuria $>3+$ on a dipstick test, urinary protein-creatinine ratio $(\mathrm{UP} / \mathrm{C})>0.2$ $\mathrm{g} / \mathrm{mmol}$ or $40 \mathrm{mg} / \mathrm{m}^{2} / \mathrm{h}$ after four weeks or more of daily corticosteroid use. Trials involving patients experiencing steroid-sensitive nephrotic syndrome, congenital nephrotic syndrome, and other kidney or systemic forms were excluded. Trials for which only abstracts were published (with no additional data available from other sources) were also excluded. No language restrictions were applied.

\section{Outcome measures}

The primary efficacy outcomes were number in $\mathrm{CR}$, number in PR, and total number in remission (TR). The primary safety outcome was the incidence of adverse secondary effects (ASEs). We defined CR as edema-free and urine protein was $<1+$ on dipstick tests, urinary UP/C $<0.02 \mathrm{~g} / \mathrm{mmol}$ or $4 \mathrm{mg} / \mathrm{m}^{2} / \mathrm{h}$ for three or more consecutive days. We defined PR as proteinuria $<2+$, urinary UP/C $<0.2 \mathrm{~g} / \mathrm{mmol}$ or $40 \mathrm{mg} / \mathrm{m}^{2} / \mathrm{h}$. TR was defined as the total number in remission, including patients in CR or PR. We defined ASEs as sequelae occurring within the initial posttreatment period (determined by each study's authors).

\section{Data extraction and risk of bias assessment}

Two investigators (SL and LT) reviewed all of the abstracts and references, evaluated the integrity of data abstraction, and rated the quality of included studies independently. We sent emails to the authors of articles with incomplete information and asked them to provide supplemental data. Methodological quality and risk of bias were assessed with the Cochrane risk of bias tool.

\section{Statistical analysis}

All data analyses were conducted in Stata, version 14 software (Stata Corp, College Station, TX, USA). First, we performed pairwise random-effects metaanalyses using the Knapp-Hartung method with the metan command, from which we report odds ratios (ORs) and 95\% confidence intervals (CIs) [39]. When a zero event was reported in a trial, the Haldane method was used to add 0.5 to each arm. We conducted traditional pairwise comparisons using the DerSimonian-Laird random effects model, which discerns and anchors trials as a sample of all potential trials. The $\mathrm{I}^{2}$ statistic was obtained as an index of heterogeneity.

Second, we conducted a network meta-analyses within a frequentist framework using the Stata network suite [40]. A multivariate random-effects meta-analysis (mvmeta command) was performed with the assumption that heterogeneity variance was consistent across treatment contrasts. We used the netleague command to report relative treatment effects for all pairwise comparisons estimated by the network meta-analysis. $P<0.05$ was considered significant. We looked at a plausible range for population difference magnitude. We used the network rank option to evaluate the probability that each drug could be the most (or second most, third most, etc.) efficacious treatment. The surface under the cumulative ranking curve (SUCRA) was determined as an estimation of the ranking probability for each medication, and the resultant SUCRA estimates were used to rank the treatments in a hierarchy [41]. We ranked the medications' safety with the same method. Within the networks, we assessed consistency between direct and indirect evidence using the design-bytreatment interaction model [42]. A loop-specific approach was applied to detect local inconsistencies within network meta-analysis models if information was sufficiently similar across sources to be combined. Difference (inconsistency factor) between direct and indirect estimations for a specific comparison was calculated with $95 \%$ CIs as a measure of within-loop inconsistency [43]. Inconsistency was defined as disagreement between direct and indirect evidence with a 95\% CI excluding 0. Publication bias was estimated by comparison-adjusted funnel plots.

\section{Author contributions}

All the authors have accepted responsibility for the full contents of this submitted manuscript and approved submission. SL and LT conceived and designed the study. SL and LT wrote the protocol. HY and QL designed and implemented the search strategies. SL and XA selected studies, assessed validity, and extracted data. JW and PG entered and analyzed the data. All authors were involved in interpreting the results, contributed to the preparation of the full review and its revision, and approved the submission of this manuscript.

\section{Abbreviations}

SRNS: steroid-resistant nephrotic syndrome; RCT: randomized controlled trial; I.v.: intravenous; MCD: minimal-change disease; MesPGN: mesangial proliferative glomerulonephritis; FSGS: focal segmental glomerulosclerosis; P/NT: placebo/nontreatment; MMF: mycophenolate mofetil; CR: complete remission; PR: partial remission; TR: total number in remission; OR: odds ratio; NS: nephrotic syndrome; CI: confidence interval; UP/C: protein-creatinine ratio; ASE: adverse secondary effect; SUCRA: surface under the cumulative ranking curve.

\section{CONFLICTS OF INTEREST}

The authors declare no conflicts of interest concerning this article. 


\section{REFERENCES}

1. McKinney PA, Feltbower RG, Brocklebank JT, Fitzpatrick MM. Time trends and ethnic patterns of childhood nephrotic syndrome in Yorkshire, UK. Pediatr Nephrol. 2001; 16:1040-1044.

2. Koskimies O, Vilska J, Rapola J, Hallman N. Long-term outcome of primary nephrotic syndrome. Arch Dis Child. 1982; 57:544-548.

3. Beck L, Bomback AS, Choi MJ, Holzman LB, Langford C, Mariani LH, Somers MJ, Trachtman H, Waldman M. KDOQI US commentary on the 2012 KDIGO clinical practice guideline for glomerulonephritis. Am J Kidney Dis. $2013 ; 62: 403-441$.

4. Prospective, controlled trial of cyclophosphamide therapy in children with nephrotic syndrome. Report of the International study of Kidney Disease in Children. Lancet. 1974; 2:423-427.

5. Tune BM, Lieberman E, Mendoza SA. Steroid-resistant nephrotic focal segmental glomerulosclerosis: a treatable disease. Pediatr Nephrol. 1996; 10:772-778.

6. Gulati A, Sinha A, Gupta A, Kanitkar M, Sreenivas V, Sharma J, Mantan M, Agarwal I, Dinda AK, Hari P, Bagga A. Treatment with tacrolimus and prednisolone is preferable to intravenous cyclophosphamide as the initial therapy for children with steroid-resistant nephrotic syndrome. Kidney Int. 2012; 1130-1135.

7. Tarshish P, Tobin JN, Bernstein J, Edelmann CM Jr. Cyclophosphamide does not benefit patients with focal segmental glomerulosclerosis. A report of the International Study of Kidney Disease in Children. Pediatr Nephrol. 1996; 10:590-593.

8. Choudhry S, Bagga A, Hari P, Sharma S, Kalaivani M, Dinda A. Efficacy and safety of tacrolimus versus cyclosporine in children with steroid-resistant nephrotic syndrome: a randomized controlled trial. Am J Kidney Dis. 2009; 760-769.

9. D'Agati VD, Alster JM, Jennette JC, Thomas DB, Pullman J, Savino DA, Cohen AH, Gipson DS, Gassman JJ, Radeva MK, Moxey-Mims MM, Friedman AL, Kaskel FJ, et al. Association of histologic variants in FSGS clinical trial with presenting features and outcomes. Clin J Am Soc Nephrol. 2013; 8:399-406.

10. Niaudet P. Treatment of childhood steroid-resistant idiopathic nephrosis with a combination of cyclosporine and prednisone. French Society of Pediatric Nephrology. J Pediatr. 1994; 125:981-986.

11. Abrantes MM, Cardoso LS, Lima EM, Penido Silva JM, Diniz JS, Bambirra EA, Oliveira EA. Predictive factors of chronic kidney disease in primary focal segmental glomerulosclerosis. Pediatr Nephrol. 2006; 21:1003-1012.

12. Gipson DS, Chin H, Presler TP, Jennette C, Ferris ME, Massengill S, Gibson K, Thomas DB. Differential risk of remission and ESRD in childhood FSGS. Pediatr Nephrol. $2006 ; 21: 344-349$.
13. Metz DK, Kausman JY. Childhood nephrotic syndrome in the 21st century: What's new? J Paediatr Child Health. 2015; 51:497-504.

14. Pravitsitthikul N, Willis NS, Hodson EM, Craig JC. Non-corticosteroid immunosuppressive medications for steroid-sensitive nephrotic syndrome in children. Cochrane Database Syst Rev. 2013; 10:Cd002290.

15. Otukesh H, Hoseini R, Rahimzadeh N, Fazel M. Rituximab in the treatment of nephrotic syndrome: a systematic review. Iran J Kidney Dis. 2013; 7:249-256.

16. Hodson EM, Willis NS, Craig JC. Interventions for idiopathic steroid-resistant nephrotic syndrome in children. Cochrane Database Syst Rev. 2010: Cd003594.

17. Abramowicz M, Barnett HL, Edelmann CM Jr, Greifer I, Kobayashi O, Arneil GC, Barron BA, Gordillo PG, Hallman N, Tiddens HA. Controlled trial of azathioprine in children with nephrotic syndrome. A report for the international study of kidney disease in children. Lancet. 1970; 1:959-961.

18. Kleinknecht C, Broyer M, Gubler MC, Palcoux JB. Irreversible renal failure after indomethacin in steroidresistant nephrosis. N Engl J Med. 1980; 302:691.

19. Garin EH, Orak JK, Hiott KL, Sutherland SE. Cyclosporine therapy for steroid-resistant nephrotic syndrome. A controlled study. Am J Dis Child. 1988; 985-988.

20. Ponticelli C, Rizzoni G, Edefonti A, Altieri P, Rivolta E, Rinaldi S, Ghio L, Lusvarghi E, Gusmano R, Locatelli F, Pasquali S, Castellani A, Della Casa-Alberighi O. A randomized trial of cyclosporine in steroid-resistant idiopathic nephrotic syndrome. Kidney Int. 1993; 43:1377-1384.

21. Elhence R, Gulati S, Kher V, Gupta A, Sharma RK. Intravenous pulse cyclophosphamide - a new regime for steroid-resistant minimal change nephrotic syndrome. Pediatr Nephrol. 1994; 1-3.

22. Lieberman KV, Tejani A. A randomized double-blind placebo-controlled trial of cyclosporine in steroid-resistant idiopathic focal segmental glomerulosclerosis in children. J Am Soc Nephrol. 1996; 56-63.

23. Mantan M, Sriram CS, Hari P, Dinda A, Bagga A. Efficacy of intravenous pulse cyclophosphamide treatment versus combination of intravenous dexamethasone and oral cyclophosphamide treatment in steroid-resistant nephrotic syndrome. Pediatr Nephrol. 2008; 1495-1502.

24. Plank C, Kalb V, Hinkes B, Hildebrandt F, Gefeller O, Rascher W, Arbeitsgemeinschaft für Pädiatrische Nephrologie. Cyclosporin A is superior to cyclophosphamide in children with steroid-resistant nephrotic syndrome-a randomized controlled multicentre trial by the Arbeitsgemeinschaft für Pädiatrische Nephrologie. Pediatr Nephrol. 2008; 1483-1493.

25. Ohri A, Phatarpekar A, Ali U, Tembekar Y. Randomized controlled trial of oral versus intravenous cyclophosphamide in idiopathic steroid resistant nephrotic syndrome. Pediatr Nephrol. 2010; 25:1879.

26. Valverde S, Hernandez AM, Velasquez L, Romero B, Mendoza A, Ramon G, Medeiros M. Efficacy of prednisone- 
tacrolimus vs. prednisone - Cyclosporine in steroid-resistant nephrotic syndrome. Pediatr Nephrol. 2010; 25:1804.

27. Magnasco A, Ravani P, Edefonti A, Murer L, Ghio L, Belingheri M, Benetti E, Murtas C, Messina G, Massella L, Porcellini MG, Montagna M, Regazzi M, et al. Rituximab in children with resistant idiopathic nephrotic syndrome. J Am Soc Nephrol. 2012; 1117-1124.

28. Sinha A BA. Randomized trial on efficacy of mycophenolate mofetil versus tacrolimus in maintaining remission in children with steroid resistant nephrotic syndrome [abstract no: HI-OR05]. J Am Soc Nephrol. 2015; 26:B2.

29. Wu B, Mao J, Shen H, Fu H, Wang J, Liu A, Gu W, Shu Q, $\mathrm{Du}$ L. Triple immunosuppressive therapy in steroid-resistant nephrotic syndrome children with tacrolimus resistance or tacrolimus sensitivity but frequently relapsing. Nephrology (Carlton). 2015; 20:18-24.

30. Hodson EM, Wong SC, Willis NS, Craig JC. Interventions for idiopathic steroid-resistant nephrotic syndrome in children. Cochrane Database Syst Rev. 2016; 10:Cd003594.

31. Hodson EM, Habashy D, Craig JC. Interventions for idiopathic steroid-resistant nephrotic syndrome in children. Cochrane Database Syst Rev. 2006; Cd003594.

32. Ishikura K, Matsumoto S, Sako M, Tsuruga K, Nakanishi K, Kamei K, Saito H, Fujinaga S, Hamasaki Y, Chikamoto H, Ohtsuka Y, Komatsu Y, Ohta T, et al. Clinical practice guideline for pediatric idiopathic nephrotic syndrome 2013: medical therapy. Clin Exp Nephrol. 2015; 19:6-33.

33. Ishikura K, Ikeda M, Hattori S, Yoshikawa N, Sasaki S, Iijima K, Nakanishi K, Yata N, Honda M. Effective and safe treatment with cyclosporine in nephrotic children: a prospective, randomized multicenter trial. Kidney Int. 2008; 1167-1173.

34. Vogel SA, Azócar PM, Nazal Ch V, Salas Del CP. Treatment guidelines for the idiopathic nephrotic syndrome in children, recommendations of the Pediatric Nephrology Branch of the Chilean Pediatric Society. Rev Chil Pediatr. 2006; 77:295-303.
35. Melocoton TL, Kamil ES, Cohen AH, Fine RN. Long-term cyclosporine A treatment of steroid-resistant and steroiddependent nephrotic syndrome. Am J Kidney Dis. 1991; 18:583-588.

36. Huwiler-Müntener K JP, Junker C, Egger M. Quality of reporting of randomized trials as a measure of methodologic quality. J Am Med Assoc. 2002; 2801-2804.

37. Cravedi P, Angeletti A, Remuzzi G. New biologics in the treatment of rare glomerular diseases of childhood. Curr Opin Pharmacol. 2017; 33:27-33.

38. Coppo R, Camilla R, Porcellini MG, Peruzzi L, Gianoglio B, Amore A, Dapra V, Loiacono E, Fonsato V, Dal Canton A, Esposito C, Esposito P, Tovo PA. Saquinavir in steroiddependent and -resistant nephrotic syndrome: a pilot study. Nephrol Dial Transplant. 2012; 27:1902-1910.

39. Harbord RM HJ. Meta-regression in Stata. Stata journal. 2008; 8:493-519.

40. IR W. Multivariate random-effects meta-regression: updates to mvmeta. Stata journal. 2011; 11:255-270.

41. Salanti G AA, Ioannidis JP. Graphical methods and numerical summaries for presenting results from multipletreatment meta-analysis: an overview and tutorial. J Clin Epidemiol. 2011; 64:163-171.

42. Jackson D BJ, Rice S, White IR, Higgins JP. A designbytreatment interaction model for network meta-analysis with random inconsistency effects. Stat Med. 2014; 33:3639-3654.

43. Song F AD, Glenny AM, Deeks JJ. Validity of indirect comparison for estimating efficacy of competing interventions: empirical evidence from published metaanalyses. BMJ. 2003; 326:472. 